\title{
A primer on TCR signaling
}

Ligation of the TCR initiates multiple signaling cascades that direct the fate of thymocytes and T cells.

ctivation of T cells through the T cell antigen receptor (TCR) is essential for thymocyte development and effector T cell function. Identification of the signaling components and how their interactions are governed is essential for understanding how T cell fate 'decisions' occur. In this issue of Nature Immunology, we present four specially commissioned articles that discuss the molecular interactions that underlie TCR signaling (http://www.nature.com/ni/focus/TCRsignaling/).

When to ignore benign complexes of self peptide and major histocompatibility complex (MHC) and when to activate downstream TCR signaling cascades in response to complexes of foreign agonist peptide and MHC has posed a conundrum since the discovery that TCRs recognize peptides presented by $\mathrm{MHC}$ molecules. As few as one to ten agonist peptide ligands can trigger productive TCR signaling, but how is this achieved? Any model put forth needs to explain the exquisite sensitivity and selectivity of TCRmediated recognition of agonist peptide-MHC complexes. Chakraborty and Weiss critically review existing models that have been proposed to explain the initiation of TCR signaling pathways. They present the strengths and weaknesses of models that invoke kinetic 'proofreading' or segregation, sequestration of immunoreceptor tyrosine-based activation motifs, allosteric malleability and serial triggering. In their analysis, they highlight the roles of the tyrosine kinases Lck and Zap70 and their interactions with subunits of the invariant signaling protein CD3 complex and the coreceptors CD4 and $\mathrm{CD} 8$, as well as the regulatory molecules that modulate these interactions. The formation of TCR microclusters is also important, as these assemblies allow signal amplification to be triggered by a single TCR-agonist peptideMHC interaction. The authors propose a new model for the initiation of TCR signaling by invoking basal phosphorylation of CD3, which poises Zap70 for activation by Lck upon interaction of the TCR with agonist peptide-MHC complexes and further stabilizes active Lck, thereby promoting the signal amplification that accounts for TCR sensitivity.

Navarro and Cantrell discuss how serine-threonine kinases transduce membrane-proximal TCR-initiated signals to elicit cytosolic and nuclear responses. TCR signaling triggers production of the second messengers $\mathrm{Ca}^{2+}$, diacylglycerol and phosphatidylinositol- $(3,4,5)$-trisphosphate, which facilitates activation of the kinases PKC, PDK1 and Akt, each a critical node for the signaling cascades that influence the activity of transcription factors that regulate gene expression in T cells. These downstream kinases coordinate the cellular response that underlies the proliferation, cytokine expression and effector function of $\mathrm{T}$ cells. Metabolic reprogramming requires activity of the kinase Erk and the metabolic checkpoint kinase complex mTORC1, which in turn activates the transcription factors c-Myc and HIF-1 and upregulation of genes encoding biosynthetic enzymes and glucose transporters. The activation of mTORC1 is also sensitive to the availability of nutrients, specifically leucine and glutamine, and the cellular ratio of AMP to ATP, which influence the inhibitory kinase AMPK. These findings might underlie the various metabolic programs of different $\mathrm{T}$ cell subsets.
Although the kinases and TCR-associated signaling molecules discussed above are relatively well known, additional molecules that influence various parameters of the ensuing cellular response continue to be found. Malissen and colleagues discuss how sensitive high-throughput mass spectrometry of affinity-purified protein complexes can be used to identify and quantify new molecular interactions. Super-resolution imaging of activated T cells also reveals information about dynamic spatial relationships between signaling components. The authors illustrate these experimental approaches with Lat, an adaptor that links TCR-initiated activation of Zap70 to multiple signaling modules, including those that effect rearrangement of the actin cytoskeleton, integrin affinity and the activation of downstream serine-threonine kinases. Notably, the kinetics of the assembly and disassembly of these molecular complexes can also be revealed by such methods. Inferences about causal relationships of protein-protein interactions or modification can subsequently be tested for functional relevance by further experimentation.

Hogquist and Jameson explore the functional consequences of TCR signaling in $\mathrm{CD}^{+} \mathrm{CD} 8^{+}$double-positive thymocytes and mature $\mathrm{T}$ cells, which are essentially 'self-reactive' to some degree, as they can respond to a diverse repertoire of peptides presented on self MHC molecules. Thymocytes undergoing negative selection or positive selection have distinct $\mathrm{Ca}^{2+}$ flux patterns in response to strong TCR signaling or weak TCR signaling, respectively. However, specialized T cell subsets, such as intraepithelial lymphocytes, invariant natural killer $\mathrm{T}$ cells and regulatory $\mathrm{T}$ cells, all require strong TCR signaling for their selection, which suggests that additional factors are needed to avoid negative selection. These processes may be influenced by the anatomical location in which the selection occurs. The sensitivity of a TCR to high- or low-affinity peptide interactions changes as cells developmentally progress from $\mathrm{CD} 4^{+} \mathrm{CD} 8^{+}$double-positive thymocytes to $\mathrm{CD} 4^{+}$or $\mathrm{CD}^{+}$single-positive thymocytes and thence to mature $\mathrm{T}$ cells and dampen their sensitivity to self peptide-MHC complexes as they exit to the periphery. This 'tuning' process is accompanied by changes in gene expression and differences in basal intracellular $\mathrm{Ca}^{2+}$ concentrations. However, basal TCR signaling in response to self peptide-MHC still occurs and may be necessary for the homeostasis of naive T cells. Intriguingly, naive $\mathrm{T}$ cell repertoires have heterogeneity in CD5 expression, which correlates with basal TCRassociated phosphorylation of $\mathrm{CD} 3 \zeta$ and may predispose mature T cells to different cell fates upon recognition of agonist peptide-MHC complexes.

It is clear that a variety of functionally specialized $\mathrm{T}$ cell subsets arise from the thymus and that the progeny of activated T cells can adopt various differentiated cell fates. Such findings suggest that additional signaling inputs beyond affinity between TCRs and peptide-MHC molecules modulate TCR signaling. The challenge for the future is to identify the molecular participants responsible and the mechanistic basis for their interactions in the framework of TCR signaling cascades. Building on this framework will allow fuller understanding of T cell responses and the regulatory mechanisms that govern adaptive immunity. 